\title{
El pensamiento crítico en estudiantes con necesidades educativas intelectuales: su desarrollo desde la musicoterapia
}

\section{Critical thinking in students with intellectual educational needs: its development from musicotherapy}

1 Ximena Cumandá Miranda López

Universidad Técnica de Ambato

xc.miranda@uta.edu.ec

2 Elsa de los Ángeles Hernández Chérrez Universidad Técnica de Ambato

eda.hernandez@uta.edu.ec

3 Déneb Sarahí Lozada Miranda

Universidad Católica del Ecuador PUCE

dslozada@puce.edu.ec

4 Christian Marcelo Lozada Miranda

Universidad Católica del Ecuador PUCESA

christian.m.lozada.m@pucesa.edu.ec

\author{
(iD https://orcid.org/0000-0001-6133-1186 \\ (D) https://orcid.org/0000-0002-6618-1559 \\ iD https://orcid.org/0000-0002-0181-4896 \\ (iD) https://orcid.org/0000-0001-5419-454X
}

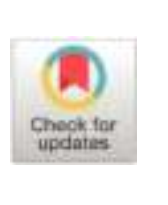

X




\section{Palabras} claves: Rey de discapacidad intelectual, pensamiento crítico, musicoterapia.
Keywords: intellectual disability,

\section{Resumen}

Introducción. El desarrollo del pensamiento crítico constituye un aspecto esencial para la vida. En los estudiantes con Necesidades Educativas Intelectuales (NEI), desarrollar el pensamiento crítico debido a las características que presentan unido a las escasas metodologías existentes para su desarrollo se convierte en un gran reto. Objetivo: reflexionar sobra la importancia de la musicoterapia como una opción para el desarrollo del pensamiento crítico en los estudiantes con Necesidades Educativas Intelectuales (NEI). Metodología: la investigación siguió una metodología descriptiva, documental, basada en la revisión sistemática de la literatura comprendida entre los años 2000 y el 2021, lográndose consultar un total 26 fuentes, de las cuales 8 constituyeron potenciales bibliográficos. Resultados: 1. se coincide en que existen diferentes concepciones sobre la definición del pensamiento crítico, así como varias metodologías para desarrollarlo; dada su complejidad e importancia en el contexto sicoeducativo, unido a los limitados estudios en el caso de los estudiantes con Necesidades Educativas Intelectuales (NEI), existe una brecha epistémica que requiere ser solucionada, de ahí que se deba seguir investigando. 2. quedó demostrada la utilidad de la musicoterapia, reafirmándose como una opción viable y efectiva para el desarrollo integral de los estudiantes con Necesidades Educativas Intelectuales (NEI), sin embargo, se hace necesario reflexionar en torno a su utilización para el desarrollo del pensamiento crítico en los estudiantes con Necesidades Educativas Intelectuales (NEI), deduciéndose que esta teoría debe ser comprobada en la práctica investigativa. 3. se propone un sistema de acciones dirigidos a desarrollar el pensamiento crítico, precisando que debe estar integrado por tres acciones básicas: diagnóstico, capacitación e intervención multidisciplinar. Conclusiones: el desarrollo del pensamiento crítico en los estudiantes con Necesidades Educativas Intelectuales es un tema de interés y altamente complejo. La Musicoterapia dada sus propias bondades y beneficios podría ser considerada una estrategia factible para el desarrollo del pensamiento crítico en los estudiantes con Necesidades Educativas Intelectuales (NEI).

Abstract

Introduction. The development of critical thinking is an essential aspect for life. In students with Intellectual Educational Needs, 
critical

thinking, music therapy developing critical thinking due to the characteristics that they present together with the few existing methodologies for their development becomes a great challenge. Target. To reflect on the importance of Music Therapy as an option for the development of critical thinking in students with Intellectual Educational Needs. Methodology. The research followed a descriptive, documentary methodology, based on the Systematic Review of the Literature between the years 2000 and 2021, managing to consult a total of 26 sources, of which 8 constituted bibliographic potentials. Results. 1.It is agreed that there are different conceptions about the definition of critical thinking, as well as various methodologies to develop it; Given its complexity and importance in the psychoeducational context, together with the limited studies in the case of students with Intellectual Educational Needs, there is an epistemic gap that needs to be solved, hence further research is needed.2. The usefulness of Music Therapy was demonstrated, reaffirming itself as a viable and effective option for the comprehensive development of students with Intellectual Educational Needs, however, it is necessary to reflect on its use for the development of critical thinking in students with Educational Needs. Intellectual Educational, inferring that this theory must be tested in investigative practice. 3. A System of actions aimed at developing critical thinking is proposed, specifying that it must be made up of three basic actions: Diagnosis, Training and Multidisciplinary Intervention. Conclusions The development of critical thinking in students with Intellectual Educational Needs is a topic of interest and highly complex. Music Therapy, given its own benefits and benefits, could be considered a feasible strategy for the development of critical thinking in students with Intellectual Educational Needs.

\section{Introducción}

El desarrollo del pensamiento crítico constituye un aspecto esencial para la vida; su optimización ayuda a tener una visión más precisa y objetiva del mundo que nos rodea. En el proceso formativo y de enseñanza-aprendizaje adquiere gran valía, esencialmente porque permite incidir positivamente en la adquisición de conocimientos, favoreciendo la formación de juicios, valoraciones, así como la toma de decisiones, lo cual interviene en la mejora del desempeño y la ganancia de habilidades teórico-prácticas en los estudiantes. Para alcanzar su desarrollo se requiere como condición básica acceder a 
formas de enseñanzas y metodologías que permitan su estimulación y a su vez se pueda contribuir a la formación integral de los estudiantes, independientemente a su condición o nivel escolar.

En el caso de los estudiantes que presentan necesidades educativas especiales de tipo intelectual, desarrollar el pensamiento crítico constituye un gran reto, precisamente por las propias características que presentan y la necesidad de provocar su desarrollo como condición indispensable para mejorar su desempeño y facilitar el proceso de inclusión educativa y social. A esto hay que agregar que ante la diversidad de estudiantes que presentan necesidades educativas de tipo intelectual, no siempre se cuenta con metodologías adecuadas que faciliten el desarrollo de su pensamiento crítico.

Dado lo anterior en el contexto educativo encontrar alternativas que motiven el desarrollo del pensamiento crítico en los estudiantes con necesidades educativas intelectuales se ha convertido en un punto de interés y reflexión para todos los educadores y personal responsabilizado con la labor formativa de estos estudiantes. En atención a lo explicado la investigación que se presenta tiene como objetivo: Reflexionar sobra la importancia de la musicoterapia como una alternativa posible a emplear para el desarrollo del pensamiento crítico en los estudiantes que presentan necesidades educativas intelectuales.

\section{Una aproximación a las necesidades educativas de tipo intelectual.}

Aproximarnos al concepto, clasificación, formas de tratamiento y escolarización de las personas con necesidades educativas especiales de tipo intelectual, también denominadas con afectaciones del intelecto o cognitivas, implicaría remontarnos a la propia existencia del hombre; básicamente porque siempre han existido personas con este tipo de condición. Lo cierto es, que en cada época histórica en el intento por conceptualizar o definir a este grupo poblacional se ha transitado por diferentes formas de denominarlos utilizando para ellos términos como: deficientes mentales, retrasados o igualmente personas con retardo en el desarrollo. Esto ha implicado hasta la actualidad el surgimiento de miradas diferentes en torno a los modos de definir y atender a este grupo poblacional que a nivel mundial alcanzan porcientos elevados.

En este orden de ideas y según informe de la Organización Mundial de la Salud (OMS, 2011), se estima que debido a múltiples factores el número de personas con algún tipo de discapacidad oscila, entre el $6 \%$ y el 10\%, de la población mundial; a ello hay que añadir que la mayoría de las personas que presentan algún tipo de discapacidad se ubica en países subdesarrollados, lo cual agrava aún más la situación que presentan en cuanto a procesos de atención de salud, escolarización, empleo, entre otros aspectos, provocándose serias afectaciones no sólo para la persona que presenta la discapacidad y para su entorno familiar y social. 
Si bien se puede considerar que el término discapacidad intelectual, ha sido definido de diferentes formas, se coindice con lo aportado en el 2011, por la Asociación Americana de Discapacidades Intelectuales y del Desarrollo (AAIDD, 2011), la cual indica que "agrupa a aquellas personas que presentan capacidades cognitivas y habilidades adaptativas disminuidas tanto en intensidad, como en los distintos grados que se presenta; lo cual puede ser perceptible desde los primeros años de sus vidas, hasta en la propia adultez". Clasificando la misma según el grado de afectación en: discapacidad intelectual: leve, moderada, grave y profunda. Es importante destacar aquí que en la generalidad de los casos tiende a presentarse antes de los 18 años.

Por su parte en el Manual de Diagnóstico y Estadística de las Trastornos Mentales (DSM5, 2015), se define a la discapacidad intelectual (trastorno del desarrollo intelectual) dentro de los trastornos del neurodesarrollo. Señalando que se trata de un grupo de afecciones cuyo inicio se sitúa en el período de desarrollo y que incluye limitaciones del funcionamiento intelectual como también del comportamiento adaptativo en cuanto a los dominios conceptuales, sociales y prácticos.

En este ámbito Peredo (2016), Flores (2018), Quimi \& Maqueira (2021), concuerdan en que la discapacidad intelectual, se ubica dentro de los problemas que más incide en el desarrollo del individuo; refieren que se caracteriza por la presencia de limitaciones significativas, bien sean de índole intelectual, como aquellas relacionadas con el desarrollo de conductas adaptativas en el individuo, afectándose la formación de conceptos, la toma de decisiones y el ajuste a las diferentes conducta y prácticas sociales, tanto el desarrollo como el aprendizaje.

En el contexto educativo se relaciona con la capacidad que está presente en el individuo para lograr adquirir los procesos de enseñanza-aprendizajes y la ganancia de hábitos, habilidades, destrezas y conductas al mismo ritmo que el resto de los estudiantes. Se enfatiza en la literatura precedente que al estar disminuida las capacidades básicas implican la necesidad de ofrecer niveles de apoyo y ayudas que pueden ser desde ayudas pedagógicas, verbales, físicas, visuales, auditivas hasta las ayudas psicológicas, las cuales contribuyen a suplir sus afectaciones cognitivas.

Al respecto Morales (2017), seguido por Quimi \& Maqueira (2021), explican que al trabajar con niños con necesidades educativas de tipo intelectual se requiere estimular tempranamente todos los procesos y áreas del desarrollo; con especial interés en todo lo relacionado con el área cognitiva, motriz, socioafectiva y del lenguaje; de esta forma se pude garantizar una mejor adaptabilidad al proceso de enseñanza-aprendizaje, asegurándose de igual manera sus procesos de inclusión. 


\section{Pensamiento crítico y discapacidad intelectual}

Analizando etimológicamente el término "pensamiento crítico", se hace necesario comprender que la palabra pensamiento, proviene del latín pensare, que significa pensar; mientras que el término crítico, se deriva del griego Kritikos, que representa juzgar, en otras palabras, está relacionado con la capacidad de comprender, discernir, descifrar, entender, imaginar; es decir, es un término que conjuga ambas palabras, el cual tiene sus orígenes a mediados del siglo XX.

En el decursar histórico el estudio del pensamiento crítico ha constituido un tema de constante debate y reflexión, tal es así que desde 1987, la fundación para el pensamiento crítico radicada en los EE.UU., mediante el consejo nacional para la excelencia en el pensamiento crítico, Ramos (2019), lo definen como:

El proceso intelectualmente disciplinado de conceptualizar, aplicar, analizar, sintetizar y / o evaluar de manera activa y hábil la información recopilada de, o por, observación, experiencia, reflexión, razonamiento o comunicación, como una guía para creer y actuar. Se basa en valores intelectuales universales tales como: claridad, precisión, consistencia, relevancia, evidencia sólida, buenas razones, profundidad, amplitud y equidad.

En esta misma línea, múltiples son los estudios que han venido subrayando la importancia del pensamiento crítico. Entre el año 2000 y el 2022, se destacan diferentes autores, los cuales han realizado significativas contribuciones relacionadas con este tema, autores como: Campos (2007), Saiz \& Rivas (2008), Saiz \& Fernández (2012), López (2012), Cabrera (2014), Morales (2014), Cárdenas et al. (2015), Coka (2017), Orellana \& Pulla (2021) y recientemente estudios realizados por Chávez (2022), destacan su utilidad en el contexto educativo, social e incluso en el área de la salud.

Al tener un acercamiento a la definición, llama la atención los aportes de López (2012), quien sostiene que los diferentes estudios sobre este tema nos inducen a pensar que se trata de algo muy controversial, en el cual han existido diferentes puntos de vistas e interpretaciones. Señalando que el pensamiento crítico, es una actividad reflexiva; en la cual el sujeto es capaz de analizar los resultados de su propia reflexión, como también los resultados derivados de la reflexión de otras personas. Destaca que se trata de un pensamiento dirigido a la acción y a la solución de problemas.

Según Morales (2014), es necesario resaltar en el hecho de incorporar el desarrollo del pensamiento crítico como parte de la teoría educativa y de la pedagogía crítica; explica que su incorporación permite estimular la formación integral del individuo desde la base del desarrollo del razonamiento, la toma de decisiones y la responsabilidad.

Por su parte Coka (2017), explica que: 
El pensamiento crítico en la educación es un instrumento para la formación integral del ser humano, por eso el docente fija reglas, determina temas, selecciona recursos, forma equipos de trabajo con el objetivo de cultivar el pensamiento crítico como un proceso donde induce a través de las actividades planificadas a que los estudiantes se identifiquen como seres humanos pensantes, listos a resolver problemas o entregar alternativas de solución como propuesta. (p.36)

Desde la década de 1980, el pensamiento crítico ha sido progresivamente reconocido por agencias, organismos e investigadores como uno de los propósitos de la educación y un área de interés creciente en varias áreas y campos del conocimiento.

Orellana \& Pulla (2021), realizan una investigación cuyo objetivo se centró en la aplicación de un programa de intervención para el fomento de las habilidades del pensamiento crítico en los alumnos del noveno año de educación básica. En dicha investigación logran determinar el nivel de desarrollo que presentan los estudiantes objeto de estudio en cuanto a su pensamiento crítico. Acentuando que la utilización de la lectura vinculada con temas de la vida diaria, contribuye de forma favorable al desarrollo del pensamiento crítico en los estudiantes. Apuntan los autores que la lectura es una alternativa que favorece el análisis y la solución de problemas, de ahí la importancia de utilizarla para el desarrollo del pensamiento crítico.

Chávez (2022), aportan una estrategia de actividades dirigidas al desarrollo de la creatividad, concluyendo que las actividades propuestas independientemente de la diversidad que se manifiesta en los estudiantes con discapacidad intelectual, contribuyen positivamente al desarrollo de su pensamiento creativo; asegurando que pueden llegar a alcanzar resultados favorables y significativos para su vida.

Derivado de los aportes realizados por los autores precedentes se puede asumir que el desarrollo del pensamiento crítico reviste gran importancia en el contexto escolar, máxime si se trata de estudiantes que presentan necesidades educativas especiales de tipo intelectual. Las características que estos presentan en su desarrollo cognitivo, así como en el resto de las áreas, hacen que sean muy propensos a lentizar su aprendizaje, lo cual influye en la realización de actividades de la vida diaria y en su inclusión educativa y social y por tanto en el desarrollo del pensamiento crítico. Buscar alternativas que permitan e incidan en su desarrollo resulta fundamental.

En este sentido la búsqueda realizada devela una realidad preocupante, determinándose que resultan limitados los estudios relacionados con el desarrollo del pensamiento crítico en los estudiantes con necesidades educativas de tipo intelectuales. Lo anterior evidencia la brecha epistémica existente y por otra parte la necesidad de motivar la realización de investigaciones relacionadas con este tema, el cual reviste gran interés para el 
perfeccionamiento de los procesos académicos, así como de inclusión y atención a la diversidad de estudiantes con necesidades educativas de tipo intelectuales.

En correspondencia con lo explicado podemos señalar que el desarrollo del pensamiento crítico en los estudiantes con necesidades educativas especiales de tipo intelectual, es por ende un proceso altamente complejo, el cual implica llevar al estudiante no solo a que interiorice conceptos, definiciones, teorías, sino que además se creen las condiciones para que desarrolle la capacidad de poder entender y utilizar creadoramente los aprendizajes logrados; se trata de lograr que los estudiantes acorde a sus propias características, nivel y posibilidades puedan identificar, argumentar, realizar análisis, deducir posibles soluciones, evaluar resultados, establecer conclusiones y aplicar lo aprendido en la práctica.

En correspondencia con lo expuesto se requiere encontrar metodologías que favorezcan el desarrollo del pensamiento crítico en estos estudiantes con necesidades educativas especiales de tipo intelectual y que a su vez estimulen el progreso de los procesos de análisis, síntesis, comparación, generalización, así como de valores como la responsabilidad, la disciplina, la honestidad, tan necesarios para su inclusión activa en la sociedad y la generación de intereses por alcanzar nuevas metas y objetivos.

Sí es importante resaltar y según consideraciones de los autores de esta investigación, que, al buscar metodologías para el desarrollo del pensamiento crítico en estudiantes con necesidades educativas especiales de tipo intelectual, dada la diversidad existente, tanto en los propios estudiantes como en las metodologías y formas de desarrollar el pensamiento crítico se deben tener en cuenta aspectos tales como:

- Diagnóstico

- Nivel de afectación

- Áreas potenciadas y limitadas.

- Habilidades cognitivas logradas

- Nivel escolar

- Significatividad del proceso de enseñanza-aprendizaje

- Contexto socioeducativo y familiar

- Relación con el medio socioeducativo.

- Apoyos necesarios

- Recursos disponibles

- Intereses, aptitudes y motivaciones presentes en los estudiantes.

Todo lo anterior permitirá desarrollar una estrategia centrada en el propio estudiante, motivando así el trabajo multidisciplinar, el cual resulta fundamental para el logro de los objetivos propuestos relacionados con el desarrollo del pensamiento crítico en estos 
estudiantes. A ello se une la necesidad de potenciar un aprendizaje significativo, que permita el vínculo de la teoría con la práctica, de modo tal que cada estudiante según sus posibilidades pueda asumir y comprender la importancia de lo que se le enseña y aprende.

La musicoterapia como alternativa para el desarrollo del pensamiento crítico en estudiantes con discapacidad intelectual

Como se ha venido comentando propiciar el desarrollo integral de los estudiantes con necesidades educativas especiales de tipo intelectual, es de extrema importancia. Ello implica potenciar el máximo desarrollo posible, lo cual incluye no solo el área cognitiva, sino también el área motriz, socioafectiva y del lenguaje, como condición esencial para su inclusión educativa y social.

En este orden de ideas y según las diferentes fuentes consultadas una de las opciones más efectivas y que más contribuye al desarrollo de los estudiantes con necesidades educativas especiales de tipo intelectual es la musicoterapia. Su empleo en los procesos de atención y rehabilitación data desde los primeros años del siglo $\mathrm{XX}$, resultando ser altamente considerado, fundamentalmente por los beneficios que provoca en el restablecimiento de las funciones físicas, psíquicas y sociales de las personas con algún tipo de discapacidad.

Según explica Poch (2001), en su trabajo sobre la importancia de la musicoterapia en el área emocional de los niños, etimológicamente la palabra musicoterapia, es una forma incorrecta de traducción del inglés. Expone que el adjetivo música, antecede al substantivo terapia, indicando que lo correcto sería plantear: terapia a través de la música; señala además que la musicoterapia forma parte de las terapias creativas, junto con la Danza-Terapia, Arte-Terapia, Poesía-Terapia y Psicodrama. Significa en su el estudio que no se trata de definirla como una terapia alternativa, de ahí que sea entendida por Poch (1981), como: "la aplicación científica del arte de la música y la danza con finalidad terapéutica, para prevenir, restaurar y acrecentar la salud tanto física como mental y psíquica del ser humano, a través de la acción del musicoterapeuta”.

A juicio de Lorenzo \& Herrera (2000), la musicoterapia promueve el desarrollo de las capacidades en los estudiantes, creando un ambiente favorable para su aprendizaje, permite desarrollar su comunicación y estimula la solución y respuesta a las diferentes situaciones de la vida diaria. En esta línea de pensamientos se coincide con Ruiz (2020), al señalar que los estudiantes con necesidades educativas especiales, pueden resultar muy beneficiados por la práctica sistemática de la música, de forma tal que no solo sea para aprender un nuevo conocimiento, sino también para sentirse mejor con su propio entorno y con ellos mismos; resalta la autora en su investigación la importancia de la labor pedagógica y de la responsabilidad de los docentes para alcanzar estos objetivos. 
En este sentido Ortega et al. (2015), seguidos García \& Rubio (2020), destacan que la musicoterapia es pródigamente empleada en los procesos de intervención con las personas con discapacidad intelectual. Asientan que la misma tiene gran utilidad para la mejora del desempeño de estas personas, ejerciendo gran influencia en su calidad de vida.

Según refieren lo anterior se puede lograr precisamente porque la musicoterapia al ser utilizada con fines educativos y terapéuticos, contribuye al desarrollo emocional, psicológico y social de los estudiantes que presentan necesidades educativas de tipo intelectual, motivando en estas diversas formas de expresión y manifestación de sus necesidades e intereses. En correspondencia con lo expuesto afirman que en los últimos años se ha podido comprobar los efectos positivos que de manera general tiene la musicoterapia en las personas con discapacidad intelectual; subrayan que la música aporta al desarrollo emocional y afectivo de estos individuos, estimulando su motricidad, la integración social, así como la disminución de situaciones de ansiedad, inquietudes, miedos y algo muy importante favorece mayor equilibrio psicótico y emocional (Ortega et al., 2015; García \& Rubio, 2020).

Por su parte Cárdenas (2020), en su investigación afirma que la utilización de la Musicoterapia puede desarrollar el potencial de las personas que presentan Necesidades Educativas Intelectuales, favoreciendo la compensación de las funciones afectadas y a su vez mejora los procesos de integración interpersonal e intrapersonal. De igual manera Valle (2021), reafirma que la musicoterapia aporta al desarrollo del aprendizaje significativo, incidiendo positivamente en el desarrollo cognitivo, psicológico y motriz de los estudiantes, resultando muy útil en el caso de los estudiantes que presentan Discapacidad Intelectual Moderada, facilita el desarrollo de aprendizajes el conocimiento de sí mismo y de su entorno.

Gran importancia tiene los aportes de Del Barrio et al. (2019), seguidos por Cárdenas (2020), al sustentar que la utilización de la música en personas con necesidades educativas especial puede realizarse teniendo en cuenta tres formas diferentes: Educación musical especial, educación musical inclusiva y la Musicoterapia, la cual tiene como objetivo mejorar la calidad de la educación, utilizando el sonido y la música para favorecer la salud física, intelectual, emocional y la comunicación.

Todo lo anterior permite confirmar la idea inicial relacionada con la importancia y utilidad de la Musicoterapia en el desarrollo integral de los estudiantes con necesidades educativas intelectuales. Esto hace que se dé un espacio importante de reflexión en torno a la posibilidad de que la Musicoterapia sea considerada una posible estrategia metodológica que de salida al desarrollo del pensamiento crítico en estos estudiantes; deduciéndose la necesidad de que esta teoría deberá ser comprobada en la práctica investigativa. 
Sí consideramos importante destacar que, para poder lograr el desarrollo del pensamiento crítico en los estudiantes con necesidades educativas intelectuales, mediante la musicoterapia se debe trabajar sobre la base del desarrollo de un sistema de acciones dirigidos a integrar todos los componentes metodológicos y didácticos que permitan potenciar el desarrollo del pensamiento crítico según las posibilidades de cada estudiante.

\section{Metodología}

La investigación siguió una metodología descriptiva de carácter documental, basada en la Revisión Sistemática de la Literatura (RSL), para lo cual se estableció como rango de tiempo las investigaciones comprendidas entre el (2000 y el 2022), publicados en base de datos Google Scholar, Dialnet, Latindex, Scopus, así como trabajos de tesis de pregrado y posgrado relacionados con el tema. Se definieron previamente los conectores de búsqueda claves, dentro de estos: necesidades educativas intelectuales, pensamiento crítico y musicoterapia, resultandos determinados como criterios de inclusión los siguientes:

\section{Criterios de Inclusión}

- Investigaciones realizadas entre el 2000 y el 2021 relacionadas con el tema.

- Tesis de grado y posgrado que aporten a la investigación, tanto de carácter descriptivo como cuasiexperimental.

- Publicaciones en idioma español en base de datos Google Scholar Dialnet, Latindex, Scopus.

La calidad de la búsqueda realizada fue controlada mediante el cumplimiento de los criterios de inclusión establecidos previamente.

\section{Resultados y Discusión}

1. Se logro consultar un total de 26 fuentes, de las cuales 8 constituyeron potenciales bibliográficos, estrechamente relacionados con el tema del Pensamiento Crítico y la Musicoterapia desde la utilidad de esta para los procesos de atención de los estudiantes con Necesidades educativas especiales de tipo Intelectual.

2. De la consulta realizada se coincide con los planteamientos de López (2012), al referir que: "existen diferentes concepciones sobre lo que es el pensamiento crítico, así como varios modelos y técnicas para fomentarlo en la escuela, pero ninguno es totalmente suficiente o superior", lo que deriva según nuestro criterio en la necesidad de continuar profundizando en el tema investigado. En este orden se concuerda con Saiz \& Rivas (2008), seguidos de Saiz \& Fernández (2012), al resaltar que es muy conveniente vincular la enseñanza de las habilidades del pensamiento crítico con situaciones o problemas cotidianos permitiendo 
desarrollar la toma de conciencia sobre las limitaciones en la forma de pensar y el enfrentar los problemas.

3. Quedó sistematiza la teoría relacionada con la importancia del pensamiento crítico, en el contexto sicoeducativo, determinándose que son escasos los estudios científicos relacionados con el desarrollo del pensamiento crítico en los estudiantes con Necesidades Educativas de tipo Intelectuales, lo cual evidencia la brecha epistémica existente y por otra parte la necesidad de motivar la realización de investigaciones relacionadas con este tema, el cual reviste gran interés para el perfeccionamiento de los procesos académicos, así como de inclusión y atención a la diversidad de estudiantes con Necesidades Educativas de tipo Intelectuales.

4. Existe coincidencia con los autores precedentes: Poch (2001), Ortega et al. (2015), Lorenzo \& Herrera (2000), García \& Rubio (2020), Ruiz (2020), y Cárdenas (2020), sobre la importancia y utilidad de la musicoterapia, en el proceso de atención de los estudiantes con Necesidades Educativas de tipo Intelectuales, reafirmándose como opción viable y efectiva para su desarrollo integral. Esto hace que se dé un espacio importante de reflexión en torno a la posibilidad de que la Musicoterapia sea considerada una posible estrategia metodológica que, de salida al desarrollo del pensamiento crítico en estos estudiantes; deduciéndose la necesidad de que esta teoría deberá ser comprobada en la práctica investigativa.

5. Se realiza la propuesta de un Sistema de acciones dirigidos a potenciar el desarrollo del pensamiento crítico en los estudiantes con Necesidades Educativas de tipo Intelectuales, precisando que el mismo debe estar integrado por las siguientes acciones:

El sistema de acciones que se presenta consiste en el desarrollo de tres acciones básicas

- 1. Diagnóstico. En esta acción se debe contemplar la aplicación de diferentes técnicas e instrumentos que permitan develar la realidad existente en cuanto a: Nivel de afectación, Áreas potenciadas y limitadas, Habilidades cognitivas logradas, Nivel escolar, Nivel de aptitudes para la música, Recursos disponibles, Significatividad del proceso de enseñanza-aprendizaje, Contexto socioeducativo y familiar.

- 2. Capacitación. En esta segunda acción y sobre el resultado del diagnóstico se debe estructurar y concretar el sistema de capitación direccionado a elevar las competencias de los docentes y padres de familia, así como de todo el entorno socioeducativo para el desarrollo de estrategias metodológicas conducentes al manejo y desarrollo adecuado del pensamiento crítico mediante la musicoterapia 
en los estudiantes con Necesidades Educativas de tipo Intelectuales. Dentro de los posibles temas a tratar se debe contemplar los siguientes:

- Necesidades Educativas de tipo Intelectuales (conceptualización, clasificación, etiologías, manifestaciones clínicas, psicológicas y pedagógicas, inclusión y atención a la diversidad)

- Pensamiento Crítico (definiciones, formas de desarrollo, importancia)

- Musicoterapia. (educación musical especial, educación musical inclusiva, componentes, musicoterapia, principales manifestaciones, orientaciones metodológicas, actividades para su desarrollo, importancia, utilidad en el contexto de los estudiantes con necesidades educativas de tipo intelectuales)

- 3. Intervención multidisciplinar. Esta acción implica un abordaje integral y multidisciplinar para el desarrollo adecuado del pensamiento crítico mediante la Musicoterapia en los estudiantes con Necesidades Educativas de tipo Intelectuales. En esta acción es importante resaltar la utilidad de las actividades lúdicas en vínculo directo con la musicoterapia de modo tal que los estudiantes aprendan de forma significativa, logren realizar procesos de análisis, síntesis, comparación, generalización, desarrollen la memoria, la atención y la capacidad creadora y argumentativa, para ello pueden auxiliarse de las diferentes manifestaciones que integran la musicoterapia.

\section{Figura 1}

Sistema de acciones dirigidos a potenciar el desarrollo del pensamiento crítico en los estudiantes con necesidades educativas de tipo intelectuales

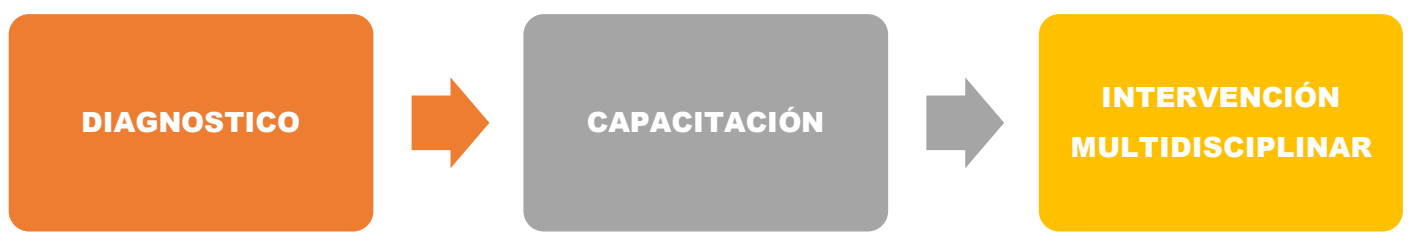

\section{Conclusiones}

- El desarrollo del pensamiento crítico en los estudiantes sigue constituyendo un tema de gran interés y altamente complejo en el caso de los que presentan Necesidades Educativas Intelectuales; de ahí la necesidad de continuar profundizando en su estudio, así como en la búsqueda de posibles soluciones que tributen a acortar las brechas epistémicas existente y a su vez permitan potenciar el desarrollo de alternativas metodológicas y acciones que posibiliten el logro del 
máximo desempeño de estos estudiantes, mediante el progreso de su pensamiento crítico.

- La Musicoterapia dada sus propias bondades y beneficios aplicada de forma organizada y en correspondencia con los interés y aptitudes de los estudiantes podría ser considerada una estrategia metodológica factible para contribuir al desarrollo del pensamiento crítico en los estudiantes con Necesidades Educativas Intelectuales.

\section{Referencias Bibliográficas}

Asociación Americana de Discapacidades Intelectuales y del Desarrollo (AAIDD). (2011). Discapacidad intelectual. Definición, clasificación y sistemas de apoyo. Alianza Editorial, Madrid. Revista Ciencias de la Salud, vol. 14, núm. 1, 2016, pp. 115-121. Universidad del Rosario Bogotá, Colombia.

Cabrera, J. (2014). Pensamiento crítico y la sociedad actual. sitiocero.

Cárdenas, L., Monroy, A., Arana, B., García, M. L., (2015), Importancia del pensamiento reflexivo y crítico en enfermería Revista Mexicana de Enfermería, Cardiológica Vol. 23, Núm. 1 enero-abril 2015, pp. 35-41

Cárdenas, R., (2020), La Musicoterapia en personas con discapacidad intelectual: Un proyecto de intervención como estrategia didáctica para el desarrollo de la autoestima. (Tesis de Maestría). Universidad de Jaén.

Campos, A. (2007). Pensamiento Crítico. Técnicas para su desarrollo. Bogotá: Cooperativa Editorial Magisterio.

Coka, J. E. (2017). Pensamiento crítico como estrategia de aprendizaje de los estudiantes con discapacidad visual en la Universidad Estatal de Milagro y el nivel de rendimiento académico. (Tesis Doctoral). Universidad Nacional Mayor de San Marcos Universidad del Perú. Lima, Perú.

Del Barrio, L. Sabbatella, P.L. \& Brotons, M.M. (2019), Musicoterapia en educación: un proyecto de innovación orientado a la inclusión del alumnado con necesidades especiales. Revista Música Hodie. Vol. (19)

Chávez, L. (2022). Estrategias para la estimulación del pensamiento crítico en estudiantes con discapacidad intelectual. Revista Innova Educación www.revistainnovaeducacion.com ISSN: 2664-1496 ISSN-L: 2664-1488 https://doi.org/10.35622/j.rie.2022.01.01 Rev. Innova Educ. (2022). Vol. 4 Núm. 1 
Flores, J. (2018)- La comprensión actual de la discapacidad intelectual. Sal Terrae | 106 (2018) 479-492

García, B., \& Rubio, C. (2020). Impacto de la musicoterapia en la calidad de vida de personas con discapacidad intelectual: una revisión de la literatura. Revista De Investigación En Musicoterapia, 3, 34-53. https://doi.org/10.15366/rim2019.3.003

López, G.A. (2012), Pensamiento crítico en el aula. Docencia e Investigación, Año XXXVII Enero/Diciembre, 2012 ISSN: 1133-9926 / e-ISSN: 2340-2725, Número 22, pp. 41-60 (Universidad Autónoma del Estado de Morelos

Lorenzo, O. \& Herrera, L. (2000). Fundamentos de Educación Musical en Educación Especial. En Música y Educación, 43, 3, (pp.65-72)

Manual de Diagnóstico y Estadística de las Trastornos Mentales [DSM-5]. (2015). A.P.A., $5^{\text {a }}$ edición. (https://www.inteligencialimite.org/discapacidad/intelectual)

Morales, L.C. (2014). El pensamiento crítico en la teoría educativa contemporánea. Revista Electrónica "Actualidades Investigativas en Educación", vol. 14, núm. 2, mayo-agosto, 2014, pp. 1-23 Universidad de Costa Rica.

Morales, E. (2017). Estimulación cognitiva y la lecto-escritura de los estudiantes de tercer año de Educación General Básica de la Unidad Educativa Julio Enrique Fernández, parroquia Izamba del Cantón Ambato. Carrera de Psicología Educativa. Universidad Técnica de Ambato. Ecuador

Orellana, D. C. \& Pulla, C. J. (2021). Programa para fomentar habilidades del pensamiento crítico en los alumnos del noveno año de Educación General Básica de la Unidad Educativa Ricardo Muñoz Chávez. (Tesis de Licenciatura). Universidad Nacional de Educación (UNAE), Carrera de Educación Básica

Organización Mundial de la Salud [OMS]. (2011). Informe mundial sobre la discapacidad.

Ortega, E., Esteban, L., Estévez, A. \& Alonso, D. (2015). Aplicaciones de la musicoterapia en educación especial y en los hospitales. European Journal of Education and Psychology, 2(2). doi:10.30552/ejep.v2i2.22

Peredo, R. A., (2016), Comprendiendo la discapacidad intelectual: Datos, criterios y reflexiones. Revista de Investigación Psicológica, (15), 101-122. http://www.scielo.org.bo/scielo.php?script=sci_arttext\&pid=S222330322016000100007\&lng=es\&tlng=es. 
Poch, S. (2001). Importancia de la Musicoterapia en el área emocional del ser humano. Revista Interuniversitaria de Formación del Profesorado, núm. 42, diciembre, 2001, pp. 91-113 Universidad de Zaragoza

Poch, S. (1981). «Musicoterapia» En: Boletín de la Sociedad Española de Pedagogía Musical». N. ${ }^{\circ}$ 2. Madrid, 29 y en «La música como vehículo de salud» en «La parella avui i altres escrits». La Llar del Llibre. Barcelona, 1988, 63-103.

Quimi Quimi, J. M., \& Maqueira, G. (2021). La estimulación cognitiva. Base para el proceso de la lectoescritura en estudiantes con discapacidad intelectual. AlfaPublicaciones, 3(3.2), 69-80. https://doi.org/10.33262/ap.v3i3.2.100

Ramos, L. (2019), Definiendo el pensamiento crítico. http://www.criticalthinking.org/

Ruiz, M. M. (2020). La importancia de la musicoterapia en niños con discapacidad intelectual. IV Congreso Internacional de Investigación e Innovación en Educación Infantil y Primaria Universidad de Murcia. http://hdl.handle.net/10201/87459

Saiz, C. \& Fernández, S. (2012): "Pensamiento crítico y aprendizaje basado en problemas Cotidianos", en Revista de Docencia Universitaria, 10(3), pp. 325 - 346.

Saiz, C. \& Rivas, S.F. (2008), "Intervenir para transferir en pensamiento crítico", en. Praxis, 10 (13), pp. 129-149

Valle, V. (2021). Musicoterapia aplicada a la discapacidad intelectual moderada. Universidad de Valladolid. Facultad de Educación de Segovia Trabajo Fin de Grado en Educación Primaria

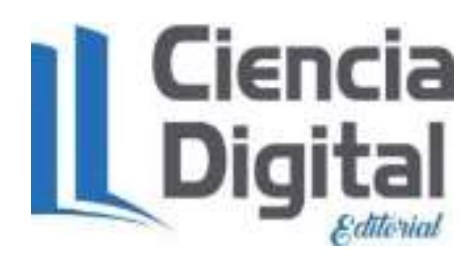


El artículo que se publica es de exclusiva responsabilidad de los autores y no necesariamente reflejan el pensamiento de la Revista Conciencia Digital.

\section{Liencia}

El artículo queda en propiedad de la revista y, por tanto, su publicación parcial y/o total en otro medio tiene que ser autorizado por el director de la Revista Conciencia Digital.
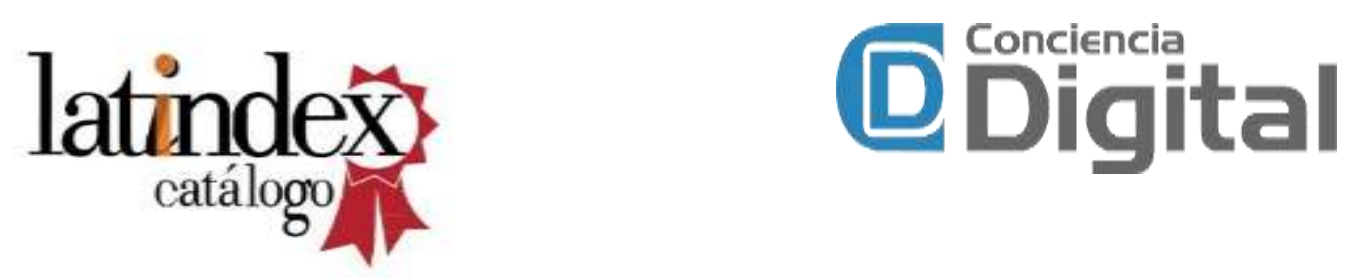

Indexaciones

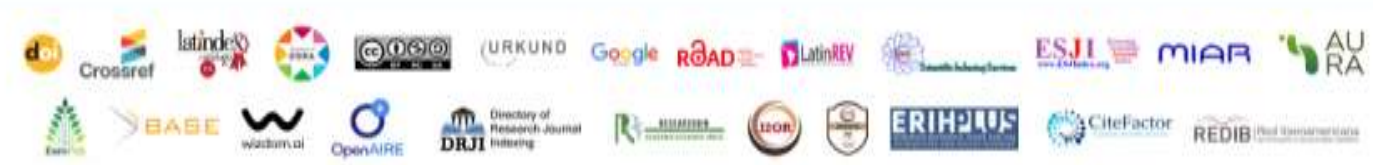

